\title{
Diet-induced gene expression of isolated pancreatic islets from a polygenic mouse model of the metabolic syndrome
}

\author{
T. Dreja $\cdot$ Z. Jovanovic $\cdot$ A. Rasche $\cdot$ R. Kluge $\cdot$ \\ R. Herwig • Y. C. L. Tung • H. G. Joost • G. S. H. Yeo • \\ H. Al-Hasani
}

Received: 6 August 2009 /Accepted: 21 September 2009 /Published online: 10 November 2009

(C) The Author(s) 2009. This article is published with open access at Springerlink.com

\begin{abstract}
Aims/hypothesis Numerous new genes have recently been identified in genome-wide association studies for type 2 diabetes. Most are highly expressed in beta cells and presumably play important roles in their function. However, these genes account for only a small proportion of total risk and there are likely to be additional candidate genes not detected by current methodology. We therefore investigated islets from the polygenic New Zealand mouse (NZL) model of diet-induced beta cell dysfunction to identify novel genes and pathways that may play a role in the pathogenesis of diabetes. Methods NZL mice were fed a diabetogenic high-fat diet (HF) or a diabetes-protective carbohydrate-free HF diet (CHF). Pancreatic islets were isolated by laser capture
\end{abstract}

T. Dreja and Z. Jovanovic contributed equally to this work, and $\mathrm{H}$. Al-Hasani and G. S. H. Yeo also contributed equally to this work.

Electronic supplementary material The online version of this article (doi:10.1007/s00125-009-1576-4) contains supplementary material, which is available to authorised users.

T. Dreja $\cdot$ R. Kluge $\cdot$ H. G. Joost $\cdot$ H. Al-Hasani $(\bowtie)$

Department of Pharmacology,

German Institute of Human Nutrition Potsdam-Rehbruecke,

Arthur-Scheunert-Allee 114-116,

14558 Nuthetal, Germany

e-mail: al-hasani@dife.de

Z. Jovanovic $\cdot$ Y. C. L. Tung $\cdot$ G. S. H. Yeo $(\bowtie)$

Institute of Metabolic Science, Level 4,

University of Cambridge Metabolic Research Laboratories,

Box 289, Addenbrooke's Hospital,

Cambridge CB2 0QQ, UK

e-mail: gshy2@cam.ac.uk

A. Rasche $\cdot$ R. Herwig

Department of Vertebrate Genomics,

Max Planck Institute for Molecular Genetics,

Berlin, Germany microdissection (LCM) and subjected to genome-wide transcriptome analyses.

Results In the prediabetic state, 2,109 islet transcripts were differentially regulated ( $>1.5$-fold) between HF and CHF diets. Of the genes identified, 39 (e.g. Cacnald, Chd2, Clip2, Igf2bp2, Dach1, Tspan8) correlated with data from the Diabetes Genetics Initiative and Wellcome Trust Case Control Consortium genome-wide scans for type 2 diabetes, thus validating our approach. HF diet induced early changes in gene expression associated with increased cellcycle progression, proliferation and differentiation of islet cells, and oxidative stress (e.g. Cdkn1b, Tmem27, Pax6, Cat, Prdx4 and Txnip). In addition, pathway analysis identified oxidative phosphorylation as the predominant gene-set that was significantly upregulated in response to the diabetogenic HF diet.

Conclusions/interpretation We demonstrated that LCM of pancreatic islet cells in combination with transcriptional profiling can be successfully used to identify novel candidate genes for diabetes. Our data strongly implicate glucose-induced oxidative stress in disease progression.

Keywords Expression profiling · Genome-wide association study - Glucotoxicity - Laser capture microdissection .

Lipotoxicity · New Zealand obese mouse · Obesity · Oxidative phosphorylation Pancreas

$\begin{array}{ll}\text { Abbreviations } \\ \text { CHF } & \begin{array}{l}\text { Carbohydrate-free high-fat diet } \\ \text { Carbohydrate-responsive element binding } \\ \text { photein }\end{array} \\ \text { DGI } & \text { Diabetes Genetics Initiative } \\ \text { GWA } & \begin{array}{l}\text { Genome-wide association study } \\ \text { HF }\end{array} \\ \text { High-fat diet } \\ \text { LCM } & \text { Laser capture microdissection }\end{array}$


NZO, NZL New Zealand obese mouse strains

OXPHOS Oxidative phosphorylation

ROS Reactive oxygen species

SNP Single nucleotide polymorphism

WTCCC Wellcome Trust Case Control Consortium

\section{Introduction}

The prevalence of type 2 diabetes and obesity has dramatically increased in recent years, driven by changes in environment and lifestyle interacting with genetic susceptibility. Recent genome-wide association studies (GWAs) in large cohorts of patients with type 2 diabetes and obesity have revealed multiple new candidate genes for these metabolic diseases [1]. Interestingly, all of the susceptibility genes for obesity strongly implicate a role for the brain, whereas most of the diabetes susceptibility genes play a role in insulin secretion and beta cell function. However, these genes explain only a small portion of the individual risk of diabetes, and it is likely that there are more candidate genes not detected by current methodology.

Inbred mouse models have been used extensively to identify genetic risk factors for obesity and type 2 diabetes $[2,3]$. Chronic hyperglycaemia is the main feature in the diabetic state, and glucotoxicity has been implicated as playing an important role in the development of type 2 diabetes [4]. Several different mechanisms have been proposed to explain the negative effects of chronic hyperglycaemia [5]. For instance, it has been proposed that elevated glucose levels lead to cellular stress, which might damage insulin-producing beta cells [6]. Nevertheless, the significance of data from transcriptional studies of islet cells is limited, since the arduous conventional collagenase digestion protocol for procuring pancreatic islets is known to alter gene expression patterns as observed in collagenaseisolated primary adipose cells [7-9]. In fact, collagenase digestion has been reported to induce a marked increase in expression of proinflammatory genes and of genes involved in hypoxia-induced stress and apoptosis, respectively, i.e. the very same pathways that have been implicated in the pathophysiology of glucose-induced beta cell death [8]. We recently introduced the New Zealand obese mouse (NZO) mouse strain as a suitable disease model for studying the role of dietary carbohydrates in the pathophysiology of high fatdiet induced diabetes [10]. In this model, a carbohydratecontaining high-fat diet (HF) is highly diabetogenic whereas a carbohydrate-free high-fat diet (CHF) protects from diabetes [10]. In the present study we isolated authentic pancreatic islets with laser capture microdissection (LCM) and performed genome-wide expression analyses with samples from HF- and CHF-treated animals. Our results support the concept that glucose-induced oxidative stress in islets is relevant for disease progression.

\section{Methods}

Experimental animals Up to six male New Zealand (NZL)/ LtJ mice (The Jackson Laboratory, Bar Harbor, MA, USA) were housed per cage (macrolon type III) at a temperature of $22^{\circ} \mathrm{C}$ with a $12 \mathrm{~h}$ light-dark cycle (lights on at 06:00 hours). The animals were kept in accordance with the NIH guidelines for the care and use of laboratory animals and all experiments were approved by the Ethics Committee of the State Ministry of Agriculture, Nutrition and Forestry (State of Brandenburg, Germany). After weaning at 3 weeks, the animals received either a HF diet (10105721; Altromin, Lage, Germany) (14.6\% [wt/wt] fat, $46.8 \%$ carbohydrates, $17.1 \%$ protein) or a $\mathrm{CHF}$ diet (10105789; Altromin) $(30.2 \%$ [wt/wt] fat, $0 \%$ carbohydrates, $26.4 \%$ protein) diet. Throughout the study the animals had free access to food and water.

Analysis of body composition Body composition and body weight were measured weekly. Body composition was measured by nuclear magnetic resonance (Echo MRI Whole Body Composition Analyzer; Echo Medical Systems, Houston, TX, USA).

Blood glucose and plasma insulin Blood glucose was measured weekly under non-fasting conditions in blood samples collected from the tail vein with a blood glucose meter (Ascensia Elite; Bayer Health Care, Leverkusen, Germany). Insulin levels were determined by ELISA (insulin mouse ultra-sensitive ELISA; DRG Instruments, Marburg, Germany).

Glucose tolerance tests At 8 weeks, animals were fasted for $16 \mathrm{~h}$ before the experiment. Each animal received a single i.p. injection of glucose $(20 \%$ solution, $2.0 \mathrm{~g} / \mathrm{kg}$ body weight). Samples for determination of blood glucose and plasma insulin concentration were collected at $0,7.5,15$, $30,60,120$ and $240 \mathrm{~min}$ from the tail tip. Mice were kept in their accustomed cage and had free access to water.

Quantitative real-time PCR Isolated islet RNA (20-50 ng) was amplified with a whole transcriptome amplification kit (TransPlex; Sigma-Aldrich, Seelze, Germany). The amplified cDNA was purified with a PCR clean-up kit (GenElute; Sigma-Aldrich). Quantitative real-time PCR was performed with the 7300 Real-Time PCR System (Applied Biosystems, Darmstadt, Germany). The PCR mix $(25 \mu \mathrm{l})$ was 
composed of TaqMan universal PCR Master Mix (10 $\mu \mathrm{l}$; Applied Biosystems), TaqMan-Probe ( $1 \mu 1$; Applied Biosystems) and $10 \mathrm{ng}$ of cDNA per reaction. Relative gene expression was calculated by the $\Delta \Delta C_{\mathrm{t}}$ method with $A c t b$ as control gene [11]. Expression differences between groups were tested with Mann-Whitney-Wilcoxon test. $p$ values below 0.05 were considered statistically significant.

Laser capture microdissection and total RNA isolation Pancreatic sections were prepared at $14 \mu \mathrm{m}$ thickness using a cryostat (Leica, Wetzlar, Germany) and mounted on RNase-free membrane-coated slides (P.A.L.M., Wolfratshausen, Germany). Immediately before microdissection, sections were fixed in ethanol and then rehydrated in $75 \%$ and $50 \%$ ethanol (vol./vol.) for $30 \mathrm{~s}$ each. Sections were stained with filtered cresyl violet acetate (Ambion) for $1 \mathrm{~min}$ to allow for identification of islets. Next, sections were dehydrated with a graded alcohol series $(50 \%, 75 \%$ and $100 \%$ and $100 \%$ (vol./vol.), $30 \mathrm{~s}$ each). Finally, the slides were placed in HistoClear (National Diagnostics, Atlanta, GA, USA) for 2 min and left to air-dry under the hood. Out of ten evenly spaced Nissl-stained sections, 500 islets were dissected using a LCM system (P.A.L.M.). Samples were stored in $20 \mu 1$ of RNAlater (Ambion). Total RNA was isolated from the LCM samples using an RNA extraction kit (RNAqueous Micro; Ambion) following the manufacturer's instructions. RNA concentrations were determined using a spectrophotometer (NanoDrop ND1000; Thermo Scientific, Wilmington, DE, USA). Quality of the RNA was determined with BioAnalyzer 2100 (Agilent, Böblingen, Germany). The RNA integrity value was used as an indicator of RNA quality. Only samples with a RNA integrity value over 4.5 were used for further analysis. Analysis of the coefficients of variation of biological replicate hybridisations confirmed homogenous RNA preparations of sufficient quality for microarray experiments (Electronic supplementary material [ESM] Fig. 1).

Amplification and labelling of LCM-isolated islet RNA For labelling of the isolated islet RNA we used an RNA amplification system (WT-Ovation Pico; NuGEN, San Carlos, CA, USA) with 100 ng RNA per reaction to produce labelled cDNA.

Hybridisation of Affymetrix microarrays For genome-wide transcriptome analysis, we used the Affymetrix Mouse Genome 430 2.0 Array platform (www.affymetrix.com/ products_services/arrays/specific/mouse430_2.affx; version 09.01.2008). LCM-isolated islets from each mouse were processed separately. Labelled cDNA $(5 \mu \mathrm{g})$ was hybridised on to each array. We analysed NZL samples from isolated islets and total pancreas with respect to two nutritional conditions induced by the HF and CHF diets.

Data processing The pre-processing and analysis of the microarray data was performed with the R/BioConductor software platform [12]. Arrays for each individual casecontrol study were normalised using the GC RMA method [13]. Affymetrix gene chip annotations were adapted from latest genome annotations in version 9, based on Ensembl 42, and resulting in 15,758 Ensembl genes [14]. Additional annotation for the Ensembl genes is based on Ensembl 46. Presence tags were calculated following Affymetrix procedures. For quality control, we analysed the coefficient of variation of the raw probe fluorescence intensities for all biological replicates in the hybridisation experiments (ESM Fig. 1). Differential expression was judged by three criteria: (1) the gene is expressed in at least one of the samples, treatment or control. at $p \leq 0.05$; (2) the fold-change is $>4 / 3$ or $<3 / 4$; and (3) the Welsh test $p$ value is $p<0.05$.

Identification of disease-related networks using enrichment analyses Disease-related networks were investigated with two different types of functional information: (1) biological pathways [15] as provided by the KEGG database (www. genome.jp/kegg/; version 09.01.2008); and (2) functional categories using Gene Ontology annotations (Ensembl 41) [16]. These functional information terms define groups of associated genes. Using enrichment analyses with hypergeometric distribution [17], we were able to assign each annotation term a $p$ value that reflects enriched occurrence of differentially expressed genes. In case of Gene Ontology terms, we included only genes with evidence codes inferred from direct assay (IDA), mutant phenotype (IMP), traceable author statement (TAS) or by curator (IC) in order to ensure a high confidence level.

Immunohistochemistry Pancreatic tissue was fixed in 4.5\% $(\mathrm{w} / \mathrm{v})$ formaldehyde and embedded in paraffin according to standard procedures. Sections $(2 \mu \mathrm{m})$ were stained with haematoxylin. Endogenous peroxidase activity in the sectioned tissues was blocked with $3 \%(v / v) \mathrm{H}_{2} \mathrm{O}_{2}$ and non-specific binding sites were blocked with non-immune goat serum. Subsequently, rabbit polyclonal antibody to carbohydrate-responsive element binding protein (ChREBP; 1:500; GeneTex, Irvine, CA, USA) was applied and stained with biotin-conjugated donkey anti-rabbit IgG and streptavidin-biotin-horseradish peroxidase complex (Dianova, Hamburg, Germany) and diaminobenzidine (Dako Diagnostika, Hamburg, Germany) as substrate according to the manufacturer's instructions. A ChREBP antibody/peptide complex (aa 800-852; NP_116569; GeneTex) served as control for non-specific binding. 


\section{Results}

A carbohydrate-free high-fat diet protects from hyperglycaemia and beta cell failure Male NZL mice were raised on two different diets, namely $\mathrm{HF}$ (35\% calories from fat) and CHF ( $72 \%$ calories from fat). As illustrated in Fig. 1a, the body weight of the animals was similar on both diets, reaching approximately $55 \mathrm{~g}$ in week 17. NZL mice also developed a similar degree of obesity on both diets, with fat depots reaching $36 \%$ of total body weight $(\sim 18 \mathrm{~g}$ total body fat; Fig. 1b). However, whereas most animals on the HF developed severe hyperglycaemia beginning at week 8 , with blood glucose levels progressively increasing, animals on the CHF maintained normal glycaemia until the end of the dietary intervention (Fig. 1c, ESM Fig. 2). At week 8, animals from both diet groups displayed severe glucose intolerance and, as described earlier [18], a markedly blunted early-phase secretion of insulin in response to a glucose bolus (Fig. 1e). At week 17, the prevalence of diabetes (blood glucose levels $>13.88 \mathrm{mmol} / \mathrm{l}$ ) in HF mice was $63 \%$. In contrast, no incidence of diabetes was observed in the CHF group (ESM Fig. 2).

Enrichment of transcripts in islets To assess the effectiveness of our islet isolation procedure, we performed quantitative real-time PCR with cDNA derived from laser-captured islets and cDNA from whole pancreas preparations. As shown in Fig. 2, the cDNAs for the islet-specific glucose transporter Slc2a2, Foxo1 and Gcg were enriched in islet preparations. To obtain a profile of global transcript abundance in islets, we compared whole-genome expression data from islets of 8-week-old CHF-fed mice with data obtained by hybridisation of RNA from total pancreas of matched animals. As expected, the list of the most abundant transcripts in islets included insulin, glucagon, islet amyloid polypeptide, carboxypeptidase $\mathrm{E}$ and other well established genes expressed in alpha and beta cells (ESM Table 1). Transcripts required for protein synthesis, protein secretion and oxidative phosphorylation were also highly abundant in the isolated islets. In contrast, the highest expression levels in total pancreas were observed for hydrolytic enzymes, including pancreatic proteases, lipases and nucleases (data not shown). To identify islet-specific transcripts, we calculated a simple enrichment score by dividing the median-normalised fluorescence intensities from islet and pancreas samples for each transcript. Of the $\sim 10,000$ genes expressed, we identified 238 transcripts that were highly enriched ( $\geq$ tenfold) in islets vs total pancreas (ESM Table 2). The list of enriched transcripts includes numerous well-known genes thought to play critical roles in islet functions such as insulin production (e.g. Cpe, Pcsk1, Pcsk2) and secretion (e.g. Abcc8, Ucn3, Glp1r, Sst, Slc2a2, Snap25), and cell growth and differen-
Fig. 1 A CHF diet protects from hyperglycaemia and diabetes. a Body weight of 17-week-old male NZL mice fed either HF or CHF. b Body fat content. c Blood glucose levels at week 17. d Plasma insulin levels at week 22 . Values were derived from 14 to 16 animals per group. e Intraperitoneal glucose tolerance test at week 8 , five animals per group. Values are mean \pm SE. White circles, CHF plasma glucose; black circles, HF plasma glucose; white squares, CHF plasma insulin; black squares, HF plasma insulin a

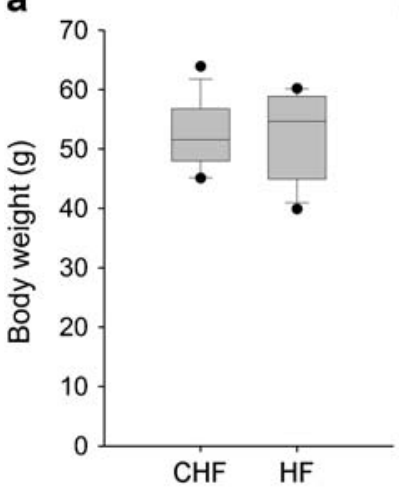

b

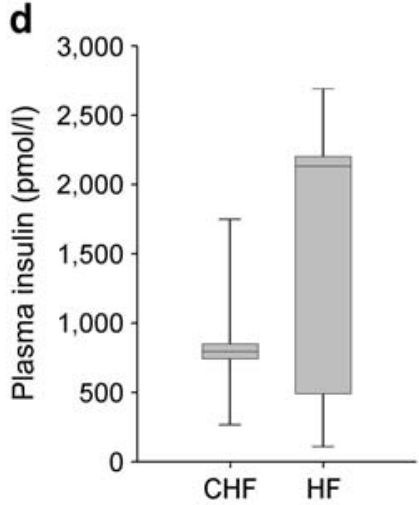

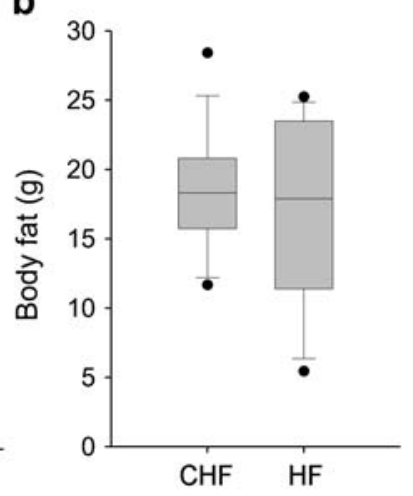

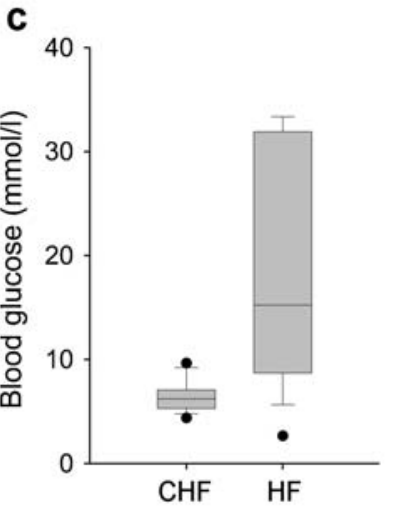

e

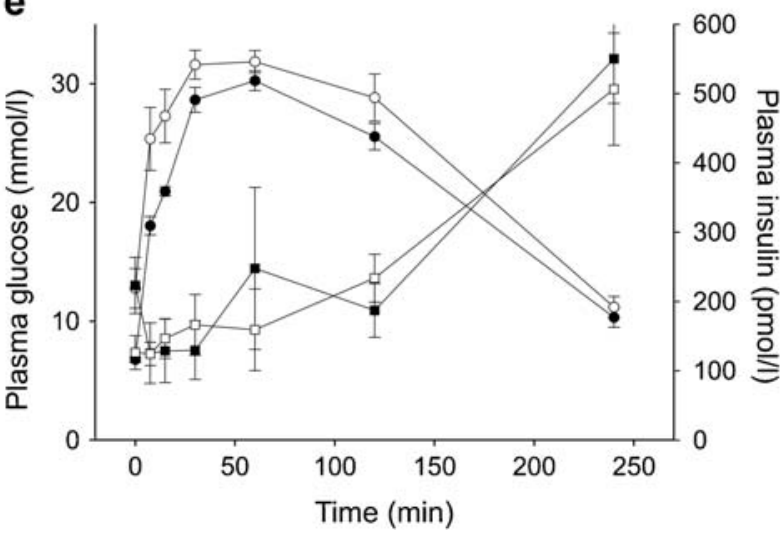




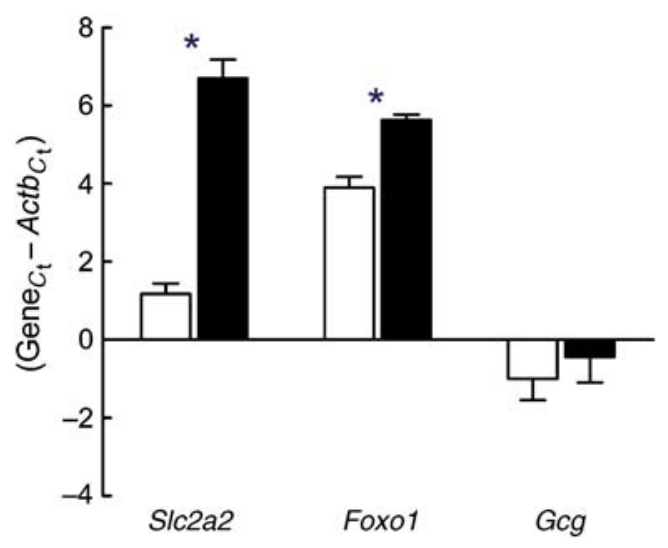

Fig. 2 Enrichment of selected islet genes in LCM samples. mRNA expression genes was determined by quantitative TaqMan RT-PCR for each gene and for Actb from LCM islets (white bars) and total pancreas (black bars) of 8-week-old mice. Expression levels are presented as change in $C_{\mathrm{t}}$ or the cycle number of each gene subtracted from the cycle number of beta-actin $\left(C_{\mathrm{t}}\right.$ value 20.15$)$ for the same cDNA sample. Lower numbers thus correspond to higher expression. Data are means \pm SE from five CHF-fed NZL mice. ${ }^{*} p \leq 0.05$

tiation (e.g. Pax6, Neurod1, Cdkn1b), as well as genes associated with type 2 diabetes in recent human genomewide scans ( $W f s$ 1, Slc30a8; ESM Table 2). Conversely, 4400 transcripts were highly enriched ( $\geq$ tenfold) in total pancreas vs islets, including the well-known pancreatic hydrolases, Cckar and members of the Reg gene family, all three of which have been implicated in islet cell regeneration (data not shown). However, expression of transcripts derived from exocrine pancreatic cells was greatly reduced in islet preparations (ESM Table 3). Yet, for a number of genes with robust islet-selective expression, the biological function is unknown.

Comparison with GWAs for type 2 diabetes Over recent years, GWAs have revealed numerous genetic loci, some of which have been implicated in the pathogenesis of diabetes, insulin secretion and beta cell survival. To determine how successful our approach was in identifying plausible novel candidates, we compared differentially expressed genes from our mouse model for diet-induced diabetes with previously reported single nucleotide polymorphisms (SNPs) associated with type 2 diabetes in humans. We used data from two publicly available genome-wide scans, the Diabetes Genetics Initiative (DGI) study [19] and the Wellcome Trust Case Control Consortium (WTCCC) GWA for diabetes [20]. We compiled a list of 837 SNPs that map to 439 known human genes and are nominally associated with diabetes $(p<0.05)$ in both scans. Of the 430 mouse orthologue genes, 39 mouse genes were differentially expressed $\geq 1.5$-fold) in our study (Table 1, ESM Fig. 3). Interestingly, the list includes two genes from genetic loci found to be associated with diabetes in recent GWAs,
IGF2BP2 [20, 21] and TSPAN8-LGR5 [22], as well as CACNA1D, which is required for pancreatic beta cell generation in mice [23]. We selected nine differentially expressed genes and performed quantitative real-time PCR with RNA from LCM-isolated islets and with RNA from total pancreas. As illustrated in ESM Fig. 4, we were able to validate all five selected genes that were downregulated by the HF, including Dach1, Clip2, Cacnald, Igf2bp2 and Chd2. In contrast, we were not able to confirm HF-induced upregulation of Ldlrad3, Sill, Plekhal and Pdia3 by quantitative real-time PCR.

Diet-dependent differentially expressed genes We identified 3,027 differentially expressed genes in islets of HF- and CHF-fed NZL mice. Of those, 2,109 genes showed at least 1.5 -fold changes in gene expression, with 1,496 genes upregulated and expression of 613 genes decreased in response to the HF. We categorised the top 50 differentially expressed genes according to their known or putative biological function and grouped these genes into the following categories of genes involved in: (1) growth and development; (2) protein processing and secretion; (3) metabolism; and (4) signalling (Table 2). In the islets, HF induced gene expression changes associated with increased cell cycle progression, proliferation and differentiation, such as Cdkn1b, Tmem 27 and Pax6 (Table 2). Also, several genes involved in insulin production and secretion were differentially regulated by the HF, such as Pcsk1 and Pcsk2 (Table 2). Moreover, known regulators of the cellular redox state such as Txnip, Cat, Gpxl and Prdx1 to Prdx5 were increased up to fourfold in the islets of HF-fed animals (Table 2). From each of the four functional categories, growth and development, protein processing and secretion, metabolism, and signalling, we selected one to two differentially expressed genes and performed quantitative real-time PCR with RNA from LCM-isolated islets and RNA from total pancreas. As illustrated in Fig. 3, dietinduced changes in expression levels for Cacnald, Cck, Mlxipl, Txnip and Tmem 27 were validated by quantitative real-time PCR in LCM islets. However, the mRNA levels for Cacnald, Mlxipl, Txnip and Tmem27 were not or only slightly changed by the diet in RNA from total pancreas, indicating that differential expression was restricted to islet cells (data not shown). Moreover, mRNA for $C c k$ (HF- $C_{\mathrm{t}}$ value in islets 25 ) was not detected in total pancreas.

Interestingly, islet mRNA for Chrebp (also known as Mlxipl), a transcription factor that has emerged as a key determinant of lipid synthesis and a major mediator of hepatic conversion of glucose into fat [24], was also significantly decreased in response to the diabetogenic HF (Table 2), a finding validated by quantitative real-time PCR (Fig. 3). Immunohistochemical staining of pancreatic sections from NZL and $\mathrm{C} 57 \mathrm{BL} / 6 \mathrm{~J}$ mice confirmed 
Table 1 Differentially expressed mouse islet genes with significant association with human type 2 diabetes in two genome-wide scans

\begin{tabular}{|c|c|c|c|c|c|c|}
\hline \multirow[t]{2}{*}{ Symbol } & \multicolumn{2}{|l|}{ Ratio } & \multirow{2}{*}{$\begin{array}{l}\text { SNPs } \\
\text { ID }^{\mathrm{c}}\end{array}$} & \multicolumn{2}{|c|}{ DGI T2D } & \multirow{2}{*}{$\begin{array}{l}\text { WTCCC T2D } \\
p \text { value }^{\mathrm{f}}\end{array}$} \\
\hline & $\mathrm{HF} / \mathrm{CHF}^{\mathrm{a}}$ & Islets/pancreas ${ }^{\mathrm{b}}$ & & $\mathrm{OR}^{\mathrm{d}}$ & $p$ value $\mathrm{e}^{\mathrm{e}}$ & \\
\hline Abcc4 & 1.789 & 1.59 & rs 1751052 & 1.179 & 0.026 & $0.018^{\mathrm{g}}$ \\
\hline \multirow[t]{9}{*}{ Arhgap 24} & 1.516 & 1.170 & rs12640411 & 0.855 & 0.027 & $0.025^{\mathrm{g}}$ \\
\hline & & & rs12506596 & 0.846 & 0.018 & $0.024^{\mathrm{g}}$ \\
\hline & & & rs 12644260 & 0.844 & 0.019 & $0.038^{\mathrm{g}}$ \\
\hline & & & rs4693716 & 0.849 & 0.023 & $0.026^{\mathrm{g}}$ \\
\hline & & & rs 1384135 & 0.842 & 0.016 & $0.027^{\mathrm{g}}$ \\
\hline & & & rs1384134 & 0.840 & 0.017 & $0.026^{\mathrm{g}}$ \\
\hline & & & rs9884932 & 0.845 & 0.018 & $0.025^{\mathrm{g}}$ \\
\hline & & & rs 10000472 & 0.845 & 0.018 & $0.026^{\mathrm{g}}$ \\
\hline & & & rs 17389300 & 0.848 & 0.020 & $0.024^{\mathrm{g}}$ \\
\hline Cacnald $^{\mathrm{h}}$ & 0.522 & 2.70 & rs 12487452 & 0.796 & 0.018 & $0.024^{\mathrm{g}}$ \\
\hline Camkk2 & 0.515 & 2.96 & rs 9805130 & 0.841 & 0.004 & $0.044^{\mathrm{g}}$ \\
\hline Car8 & 1.771 & 1.13 & rs 10216440 & 0.833 & 0.026 & $0.001^{\mathrm{g}}$ \\
\hline$C h d 2^{\mathrm{h}}$ & 0.532 & 21.00 & rs 1406712 & 1.379 & 0.039 & $0.023^{\mathrm{g}}$ \\
\hline \multirow[t]{2}{*}{ Clip $2^{\mathrm{h}}$} & 0.435 & 7.91 & rs2528994 & 1.118 & 0.020 & $0.021^{\mathrm{g}}$ \\
\hline & & & rs5 12023 & 1.098 & 0.029 & $0.042^{\mathrm{g}}$ \\
\hline Cobll1 & 2.348 & 0.19 & rs6717858 & 0.892 & 0.027 & $0.026^{\mathrm{g}}$ \\
\hline Ctnnd2 & 1.877 & 9.39 & rs6873671 & 1.138 & 0.025 & $0.034^{\mathrm{i}}$ \\
\hline \multirow[t]{2}{*}{ Esyt2 } & 1.602 & 0.47 & rs3816465 & 1.198 & 0.037 & $0.009^{\mathrm{i}}$ \\
\hline & & & rs6873490 & 1.137 & 0.019 & $0.030^{\mathrm{i}}$ \\
\hline $\operatorname{Dach} 1^{\mathrm{h}}$ & 0.352 & 8.78 & rs7991293 & 1.469 & 0.019 & $0.010^{\mathrm{i}}$ \\
\hline \multirow[t]{3}{*}{ Elf1 } & 1.855 & 0.24 & rs 9525438 & 1.143 & 0.034 & $0.018^{\mathrm{i}}$ \\
\hline & & & rs7799 & 1.143 & 0.028 & $0.005^{\mathrm{i}}$ \\
\hline & & & rs9532691 & 1.148 & 0.024 & $0.007^{\mathrm{i}}$ \\
\hline \multirow[t]{2}{*}{ Fam $45 a$} & 1.571 & 0.60 & rs 4752250 & 0.856 & 0.043 & $0.005^{\mathrm{g}}$ \\
\hline & & & rs 11198783 & 0.837 & 0.015 & $0.005^{\mathrm{g}}$ \\
\hline \multirow[t]{2}{*}{ Gnaq } & 1.541 & 0.93 & rs6560613 & 0.777 & 0.034 & $0.007^{\mathrm{g}}$ \\
\hline & & & rs4745664 & 0.839 & 0.024 & $0.040^{\mathrm{g}}$ \\
\hline$H 2-E a$ & 1.806 & 0.41 & rs7194 & 0.872 & 0.041 & $0.045^{\mathrm{g}}$ \\
\hline \multirow[t]{6}{*}{ Hnrnpc } & 1.582 & 0.87 & rs17092523 & 0.936 & 0.033 & $0.001^{\mathrm{i}}$ \\
\hline & & & rs4982437 & 0.914 & 0.021 & $0.010^{\mathrm{i}}$ \\
\hline & & & rs7495 & 0.929 & 0.028 & $0.005^{\mathrm{i}}$ \\
\hline & & & rs8009894 & 0.941 & 0.046 & $0.006^{\mathrm{i}}$ \\
\hline & & & rs 7141230 & 0.938 & 0.039 & $0.004^{\mathrm{i}}$ \\
\hline & & & rs7147655 & 0.935 & 0.036 & $0.007^{\mathrm{i}}$ \\
\hline \multirow[t]{2}{*}{$\operatorname{Ig} f 2 b p 2^{\mathrm{h}}$} & 0.493 & 5.74 & rs4402960 & 1.110 & 0.034 & $0.002^{\mathrm{g}}$ \\
\hline & & & rs7651090 & 1.103 & 0.039 & $0.002^{\mathrm{g}}$ \\
\hline \multirow[t]{3}{*}{$\operatorname{Ig} f 2 r$} & 1.904 & 1.24 & rs10945645 & 0.780 & 0.007 & $0.039^{\mathrm{i}}$ \\
\hline & & & rs3798197 & 0.869 & 0.028 & $0.032^{\mathrm{i}}$ \\
\hline & & & rs9295119 & 0.836 & 0.040 & $0.012^{\mathrm{i}}$ \\
\hline \multirow[t]{3}{*}{ Inpp $5 d$} & 0.588 & 2.51 & rs10176895 & 0.7583 & 0.043 & $0.025^{\mathrm{g}}$ \\
\hline & & & rs10189488 & 0.735 & 0.018 & $0.035^{\mathrm{g}}$ \\
\hline & & & rs10200271 & 0.7084 & 0.022 & $0.045^{\mathrm{g}}$ \\
\hline Klhl21 & 1.688 & 1.22 & rs 1556036 & 1.122 & 0.031 & $0.008^{\mathrm{i}}$ \\
\hline Ldlrad3 & 5.274 & 3.56 & rs 12361738 & 0.869 & 0.020 & $0.023^{\mathrm{g}}$ \\
\hline Lyar & 1.52 & 0.158 & rs2980089 & 1.217 & 0.016 & $0.05^{\mathrm{g}}$ \\
\hline Mphosph6 & 1.941 & 0.96 & rs 1862820 & 1.201 & 0.009 & $0.004^{\mathrm{g}}$ \\
\hline
\end{tabular}


Table 1 (continued)

\begin{tabular}{|c|c|c|c|c|c|c|}
\hline \multirow[t]{2}{*}{ Symbol } & \multicolumn{2}{|l|}{ Ratio } & \multirow{2}{*}{$\begin{array}{l}\text { SNPs } \\
\text { ID }^{\mathrm{c}}\end{array}$} & \multicolumn{2}{|c|}{ DGI T2D } & \multirow{2}{*}{$\begin{array}{l}\text { WTCCC T2D } \\
p \text { value }^{\mathrm{f}}\end{array}$} \\
\hline & $\mathrm{HF} / \mathrm{CHF}^{\mathrm{a}}$ & Islets/pancreas ${ }^{b}$ & & $\mathrm{OR}^{\mathrm{d}}$ & $p$ value $^{\mathrm{e}}$ & \\
\hline Nampt & 1.584 & 0.48 & rs4730155 & 0.909 & 0.043 & $0.012^{\mathrm{g}}$ \\
\hline Pdia3 & 3.384 & 0.50 & rs 8040336 & 0.852 & 0.012 & $0.045^{\mathrm{g}}$ \\
\hline \multirow[t]{2}{*}{ Pdlim5 } & 0.561 & 4.80 & rs6830074 & 0.804 & 0.047 & $0.003^{\mathrm{g}}$ \\
\hline & & & rs6812098 & 0.790 & 0.041 & $0.015^{\mathrm{g}}$ \\
\hline \multirow[t]{6}{*}{ Phactr1 } & 0.525 & 33.9 & rs17617584 & 0.770 & 0.027 & $0.048^{\mathrm{g}}$ \\
\hline & & & rs9296486 & 0.794 & 0.009 & $0.043^{\mathrm{g}}$ \\
\hline & & & rs9296488 & 0.799 & 0.011 & $0.032^{\mathrm{g}}$ \\
\hline & & & rs9349350 & 0.802 & 0.012 & $0.023^{\mathrm{g}}$ \\
\hline & & & rs9357503 & 0.837 & 0.033 & $0.028^{\mathrm{g}}$ \\
\hline & & & rs9381439 & 0.804 & 0.013 & $0.035^{\mathrm{g}}$ \\
\hline Ppfia3 & 0.6 & 4.41 & rs3764625 & 1.132 & 0.023 & $0.003^{\mathrm{g}}$ \\
\hline Pkib & 2.289 & 1.52 & rs 17084680 & 1.085 & 0.025 & $0.614^{\mathrm{g}}$ \\
\hline Plekhal & 2.424 & 0.51 & rs 17103541 & 1.303 & 0.023 & $0.028^{\mathrm{g}}$ \\
\hline Qpct & 3.93 & 0.52 & rs 2255991 & 1.368 & 0.029 & $0.01805^{\mathrm{g}, \mathrm{i}}$ \\
\hline Sill & 1.951 & 0.57 & rs1560656 & 0.871 & 0.022 & $0.042^{\mathrm{i}}$ \\
\hline Stxbp6 & 1.825 & 0.19 & rs 17257773 & 1.352 & 0.006 & $0.024^{\mathrm{i}}$ \\
\hline \multirow[t]{3}{*}{ Tacr3 } & 2.138 & 1.27 & rs 1384401 & 0.828 & 0.011 & $0.015^{\mathrm{g}}$ \\
\hline & & & rs5005634 & 0.835 & 0.011 & $0.011^{\mathrm{g}}$ \\
\hline & & & rs7663664 & 0.821 & 0.009 & $0.010^{\mathrm{g}}$ \\
\hline \multirow[t]{2}{*}{ Tmem 135} & 1.766 & 0.72 & rs 10898589 & 1.428 & 0.018 & $0.044^{\mathrm{g}}$ \\
\hline & & & rs 10898590 & 1.449 & 0.013 & $0.009^{\mathrm{g}}$ \\
\hline \multirow[t]{4}{*}{ Tspan 8} & 3.993 & 1.35 & rs 10506625 & 0.860 & 0.019 & $0.00007^{\mathrm{g}}$ \\
\hline & & & rs 10506626 & 1.146 & 0.015 & $0.00002^{\mathrm{g}}$ \\
\hline & & & rs11178648 & 1.158 & 0.014 & $0.00005^{\mathrm{g}}$ \\
\hline & & & rs 2270584 & 1.146 & 0.012 & $0.00002^{\mathrm{g}}$ \\
\hline \multirow[t]{4}{*}{ Ube $2 z$} & 1.759 & 0.94 & rs 15563 & 0.903 & 0.031 & $0.028^{\mathrm{i}}$ \\
\hline & & & rs 46522 & 0.906 & 0.040 & $0.025^{\mathrm{i}}$ \\
\hline & & & rs1994970 & 0.907 & 0.040 & $0.033^{\mathrm{i}}$ \\
\hline & & & rs4793992 & 0.904 & 0.043 & $0.029^{\mathrm{i}}$ \\
\hline$X k r 6$ & 0.537 & 3.11 & rs7016385 & 1.115 & 0.033 & $0.019^{\mathrm{g}}$ \\
\hline
\end{tabular}

Expression of genes was determined with mRNA from LCM islets and from total pancreas of 8-week-old mice

${ }^{a}$ Only genes with a significant fold-change $(p \leq 0.05)$ were included

${ }^{\mathrm{b}}$ Ratio of median-normalised fluorescence intensities from islet and pancreas samples for each transcript

${ }^{\mathrm{c}}$ Only SNPs with $p \leq 0.05$ in both GWAs $[19,20]$ were included

d,e Odds ratios and $p$ values for association with type 2 diabetes in DGI study

${ }^{\mathrm{f}}$ As above $\left({ }^{\mathrm{d}, \mathrm{e}}\right)$ in WTCCC Study

${ }^{\mathrm{g}}$ Additive model; ${ }^{\mathrm{h}}$ differential expression validated by quantitative real-time; ${ }^{\mathrm{i}}$ general model

presence of ChREBP protein in islet cells (Fig. 4a-d), although we were unable to quantify its nuclear localisation. According to quantitative real-time PCR analysis, expression of Chrebp mRNA was comparable in liver and islet tissue. However, only in the liver of the animals, did the HF result in substantial upregulation of $P k l r, S c d l$ and malic enzyme, whereas no significant changes in gene expression levels were observed in the corresponding islets of the animals (Fig. 4e).
Pathway analysis The set of differentially expressed genes was used to perform pathway analysis as described. Gene set enrichment of the most significant KEGG pathways revealed differential expression of genes involved in mitochondrial oxidative phosphorylation, proteasome, ribosome, pyruvate metabolism, terpenoid biosynthesis, Nglycan biosynthesis, bisphenol A degradation and reductive carboxylate cycle (Table 3, ESM Table 4). Oxidative phosphorylation was the most significantly over-represented 
Table 2 Diet-dependent differentially expressed genes in islets

\begin{tabular}{|c|c|c|}
\hline Symbol per category & Description & Ratio $(\mathrm{HF} / \mathrm{CHF})^{\mathrm{a}}$ \\
\hline \multicolumn{3}{|c|}{ Growth and development } \\
\hline Pax6 & Paired box gene 6 & 0.51 \\
\hline$C d k n 1 b$ & Cyclin-dependent kinase inhibitor 1B & 0.40 \\
\hline Pura & Purine rich element binding protein A & 0.37 \\
\hline Dach1 & Dachshund 1 (Drosophila) & 0.35 \\
\hline \multicolumn{3}{|l|}{ Metabolism } \\
\hline Gmds & GDP-mannose 4, 6-dehydratase & 5.02 \\
\hline Ndufal & NADH dehydrogenase (ubiquinone) 1 alpha subcomplex, 1 & 4.80 \\
\hline Sqle & Squalene epoxidase & 4.64 \\
\hline Atp $5 b$ & ATP synthase, $\mathrm{H}+$ transporting mitochondrial $\mathrm{F} 1$ complex, beta subunit & 4.40 \\
\hline Prps1 & Phosphoribosyl pyrophosphate synthetase 1 & 4.23 \\
\hline Suclg1 & Succinate-CoA ligase, GDP-forming, alpha subunit & 4.14 \\
\hline Pon3 & Paraoxonase 3 & 4.13 \\
\hline Scdl & Stearoyl-Coenzyme A desaturase 1 & 2.80 \\
\hline$P f k f b 2$ & 6-Phosphofructo-2-kinase/fructose-2,6-biphosphatase 2 & 0.41 \\
\hline Cyp4a10 & Cytochrome P450, family 4 , subfamily $a$, polypeptide 10 & 0.35 \\
\hline Chkb & Choline kinase beta & 0.34 \\
\hline Eno3 & Enolase 3 , beta muscle & 0.34 \\
\hline Mlxipl & MLX interacting protein-like & 0.29 \\
\hline Gmds & GDP-mannose 4, 6-dehydratase & 0.20 \\
\hline \multicolumn{3}{|c|}{ Protein processing and secretion } \\
\hline Rps $27 l$ & Ribosomal protein S27-like & 5.05 \\
\hline Psmb7 & Proteasome (prosome, macropain) subunit, beta type 7 & 4.85 \\
\hline Vamp8 & Vesicle-associated membrane protein 8 & 3.15 \\
\hline Pcskl & Proprotein convertase subtilisin/kexin type 1 & 2.73 \\
\hline Pcsk2 & Proprotein convertase subtilisin/kexin type 2 & 1.92 \\
\hline$V t i 1 b$ & Vesicle transport through interaction with t-SNAREs 1B homologue & 1.88 \\
\hline Vamp5 & Vesicle-associated membrane protein 5 & 0.40 \\
\hline $\operatorname{Sec} 24 a$ & SEC24 related gene family, member A (S. cerevisiae) & 0.38 \\
\hline \multicolumn{3}{|l|}{ Signalling } \\
\hline Cck & Cholecystokinin & 9.25 \\
\hline Rheb & RAS-homologue enriched in brain & 4.82 \\
\hline Dusp6 & Dual specificity phosphatase 6 & 4.26 \\
\hline Cacnald & Calcium channel, voltage-dependent, L type, alpha 1D subunit & 0.52 \\
\hline Rps6kb2 & Ribosomal protein S6 kinase, polypeptide 2 & 0.40 \\
\hline Nlgn1 & Neuroligin 1 & 0.34 \\
\hline Viprl & Vasoactive intestinal peptide receptor 1 & 0.33 \\
\hline P2ry6 & Pyrimidinergic receptor P2Y, G-protein coupled, 6 & 0.28 \\
\hline \multicolumn{3}{|c|}{ Regulation of redox status } \\
\hline Txnip & Thioredoxin interacting protein & 4.14 \\
\hline $\operatorname{Prd} x 4$ & Peroxiredoxin 4 & 3.10 \\
\hline $\operatorname{Prdx} 5$ & Peroxiredoxin 4 & 2.74 \\
\hline Cat & Catalase & 1.95 \\
\hline Gpxl & Glutathione peroxidase 1 & 2.26 \\
\hline \multicolumn{3}{|l|}{ Other } \\
\hline Ctss & Cathepsin S & 12.98 \\
\hline Cldn11 & Claudin 11 & 11.32 \\
\hline Sppl & Secreted phosphoprotein 1 & 4.55 \\
\hline
\end{tabular}


Table 2 (continued)

Symbol per category

\begin{tabular}{lll}
\hline Alg5 & Asparagine-linked glycosylation 5 homolog & 4.54 \\
Tmem 27 & Transmembrane protein 27 & 1.94
\end{tabular}

The top 44 differentially expressed genes (HF vs CHF) were categorised according to their known or putative biological function

${ }^{a}$ Mean of five to six Affymetrix chips per condition; only genes with a significant fold-change were included $(p \leq 0.05)$

pathway $(2.31 \mathrm{E}-12)$ whose expression was differentially regulated between HF and CHF. About half of the genes annotated for oxidative phosphorylation (OXPHOS), proteasome and ribosome were differentially expressed in islets in response to the dietary regiment. In fact, the majority of these genes annotated for these three pathways were upregulated in response to HF (Table 3). To confirm this observation, we performed quantitative real-time PCR on six OXPHOS genes that are derived from complex I (Ndufa8, Ndufb 9 and $N d u f c 2$ ) and on one from each of complexes II, III and IV (Sdhd, Uqcrc2 and Cox5a, respectively). With the exception of Uqcrcl from complex III, each transcript was upregulated in response to HF, with significant changes for $N d u f a 8, N d u f b 9$ and $N d u f c 2$ (Fig. 5).

\section{Discussion}

To identify potentially relevant candidate genes involved in type 2 diabetes, we used a model of beta cell failure, NZL mice, fed either a HF or a CHF diet. To gain insights into

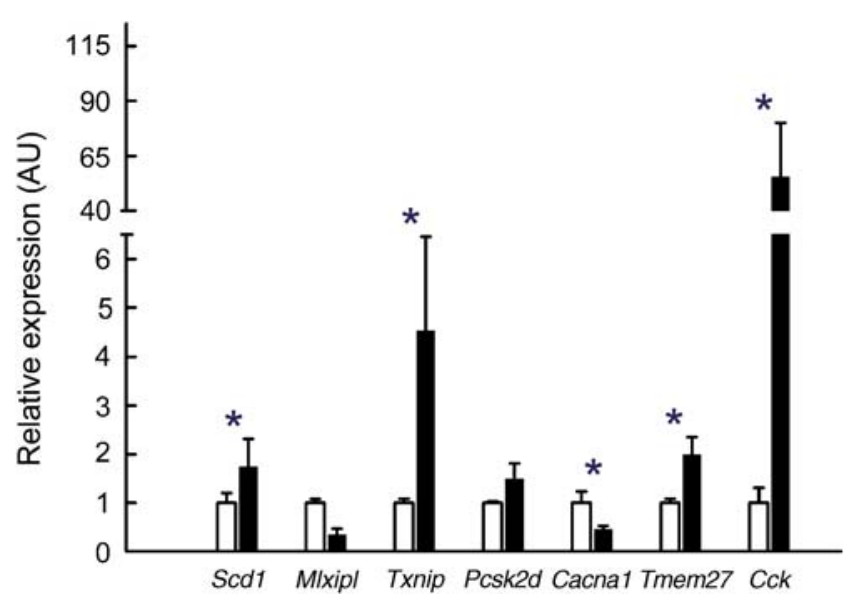

Fig. 3 Validation of differential expression in islets by quantitative real-time PCR. TaqMan quantitative PCR validation of genes differentially regulated between CHF (white bars) and HF (black bars). The change in expression is shown for selected genes involved in islet growth and development, protein processing and secretion, metabolism, and signalling. Values are normalised to Actb. Data are mean \pm SE of five separate mice per group. ${ }^{*} p \leq 0.05$ the pathophysiology of diet-induced beta cell failure and the metabolic pathways mediating downstream effects of the HF, we isolated pancreatic islets with LCM and performed genome-wide expression analyses. Consistent with previous findings $[10,25]$, our data clearly demonstrate the critical
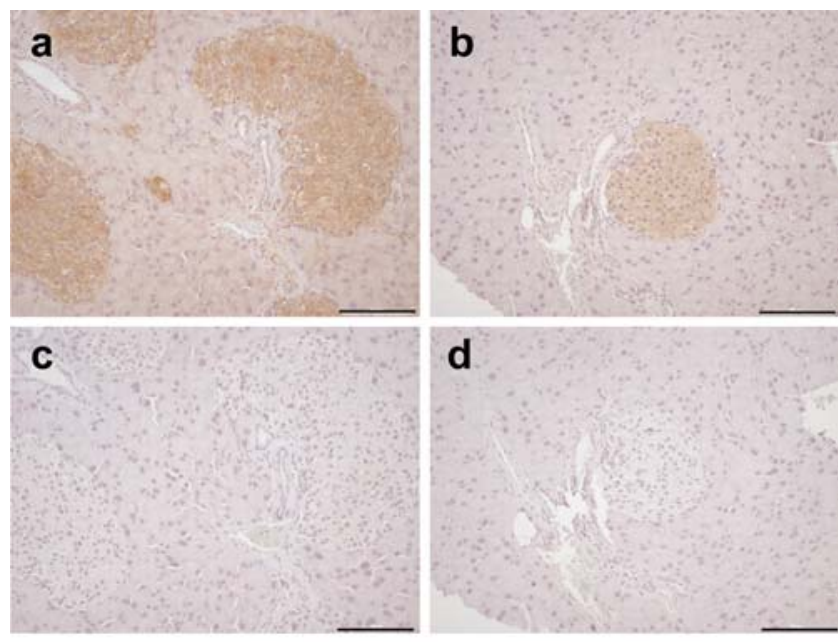

d

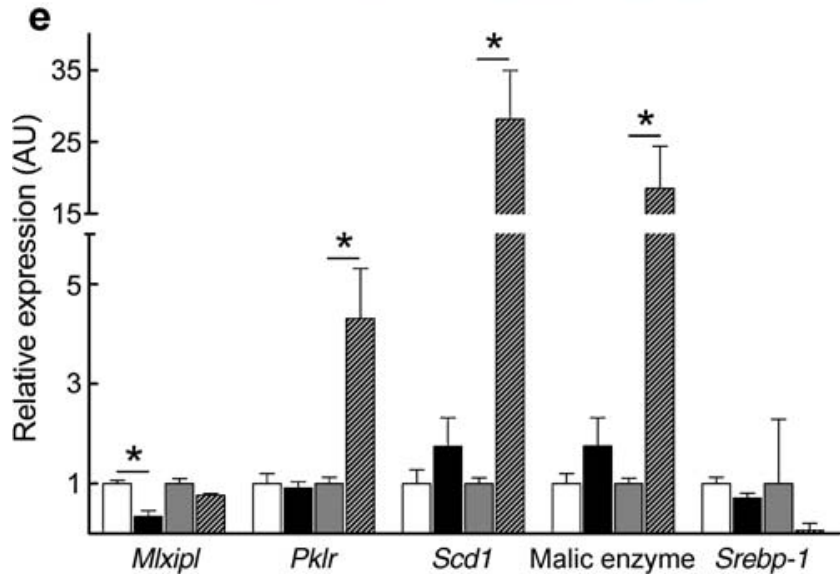

Fig. 4 Islet levels of ChREBP and regulation of lipogenic target genes. Immunohistochemical detection of ChREBP in pancreatic sections from male NZL mice. HF (a) and CHF fed (b) animals at week 8 , with (c, d) respective controls with blocking peptide. e TaqMan quantitative PCR validation of ChREBP target genes in islets. Values are normalised to Actb. Data are mean $\pm \mathrm{SE}$ of five separate mice per group; ${ }^{*} p \leq 0.05$. White bars, CHF; black bars, HF; grey bars, CHF liver; hatched bars, HF liver. Srebp-1 is also known as Srebf1 
Table 3 Gene set enrichment analysis of 3,027 genes differentially regulated by HF and/or CHF diet in islets

\begin{tabular}{llcccc}
\hline KEGG pathway description & $p$ value & Set $^{\mathrm{a}}$ & Sig.Set $^{\mathrm{b}}$ & Sig.Set, upregulated $^{\mathrm{b}}$ & Sig.Set, downregulated $^{\mathrm{b}}$ \\
\hline Oxidative phosphorylation & $2.31 \times 10^{-12}$ & 96 & 49 & 46 & 3 \\
Proteasome & $1.41 \times 10^{-7}$ & 25 & 17 & 17 & 0 \\
Ribosome & 0.00297 & 21 & 10 & 9 & 1 \\
Pyruvate metabolism & 0.00639 & 37 & 14 & 2 & 2 \\
Terpenoid biosynthesis & 0.00708 & 3 & 3 & 13 & 1 \\
$N$-Glycan biosynthesis & 0.00836 & 38 & 8 & 6 & 2 \\
Bisphenol A degradation & 0.0085 & 17 & 6 & 6 & 0 \\
Reductive carboxylate cycle & 0.0095 & 11 & & 1 \\
\hline
\end{tabular}

Only pathways with significant changes $(p \leq 0.01)$ were included

${ }^{a}$ Number of genes in KEGG pathway; ${ }^{\text {b }}$ differentially expressed genes

role of dietary carbohydrates in the pathophysiology of high fat-diet induced diabetes. In the obese NZL mice as well as the related NZO strain, a CHF protects from hyperglycaemia and beta cell failure, whereas a carbohydrate-containing HF diet is highly diabetogenic [10]. Since both diets induce a similar degree of adiposity and glucose intolerance, the beta cell toxicity is likely to be due to elevated glucose concentrations.

As expected, numerous genes known to be highly expressed in islets, such as insulin, glucagon and islet amyloid polypeptide, were also highly abundant in the LCM samples. In fact, about $2.5 \%$ of all transcripts expressed were highly enriched in islets cells compared with total pancreas. Moreover, many of those transcripts have not been linked to islet cell function previously and may therefore represent novel candidate genes with relevant functions in islet cell biology. Most importantly, however, islet RNA isolated with LCM is likely to reflect the transcriptome of these cells more accurately, compared with the conventional collagenase-digestion method used

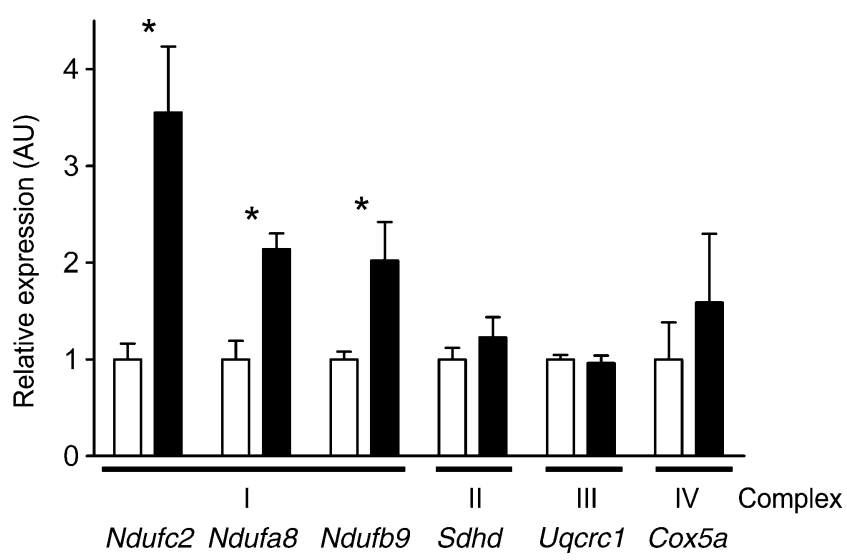

Fig. 5 TaqMan quantitative PCR validation of OXPHOS genes in islets. The changes in expression of selected OXPHOS genes are shown. Values are normalised to Actb. Data are mean $\pm \mathrm{SE}$ of five separate mice per group; ${ }^{*} p \leq 0.05$. White bars, CHF; black bars, HF for islet isolation [7, 8]. In fact, the latter procedure has been reported to induce marked increases in gene expression of proinflammatory genes and of genes involved in hypoxia-induced stress and apoptosis, thereby severely impairing interpretation of expression data derived from metabolically stressed islet cells [7, 8]. Thus, LCM represents an ideal tool to obtain native islet samples without inducing the stress-response of islet isolation.

Recent GWAs have revealed numerous genetic loci associated with type 2 diabetes in humans [1]. So far, there has been a lack of functional data about the involvement of these variants in the pathogenesis of diabetes. However, most of these genes were found to be highly expressed in islets [1]. We identified 39 genes that are highly expressed in the pancreas and were differentially expressed in our mouse model of diet-induced diabetes and nominally associated with diabetes in two independent GWAs, the DGI study and the WTCCC scan for diabetes, respectively $[19,20]$, thus validating our approach (Table 1). Among these genes is $C A C N A 1 D$, the voltage-dependent calcium channel, which has been shown to be required for pancreatic beta cell generation in mice [23]. Interestingly, the list also includes two loci that have been found to be associated with type 2 diabetes in recent GWAs, IGF2BP2 [19, 21] and TSPAN8-LGR5 [22]. With regard to the latter region, the most significant association with type 2 diabetes in a meta-analysis of three GWA scans was observed for rs7961581 $\left(p=1.1 \times 10^{-9}\right)$, which maps in the intergenic region between TSPAN8 and LGR5 [22]. In mouse islet cells, the diabetogenic HF increased expression of Tspan 8 approximately fourfold, whereas Lgr5 expression was not changed (Table 3, data not shown). Thus, our data provide evidence that TSPAN8 is more likely to be functionally related to the development of type 2 diabetes than $L G R 5$. It should be mentioned, however, that our approach has limitations, since we only took into account the two publicly available genome-wide scans on type 2 diabetes susceptibility genes. Moreover, the overlap of differentially 
expressed genes in islets and genes found in the two GWAs amounts roughly to the number expected by chance. However, this number is likely to be underestimated, because not all genes were tested for association with type 2 diabetes in the merged scans.

Exposure to HF resulted in a substantial number of differentially expressed genes in the islets, reflecting prominent changes of the expression pattern in response to the diet. In fact, the majority of these genes $(>80 \%)$ were upregulated in HF islets. We have previously shown that in the prediabetic state, HF induces massive hyperglycaemia in the related NZO strain and compensatory hyperplasia of pancreatic islets $[10,26,27]$. Consistent with these data, HF induced gene expression changes associated with increased islet growth and insulin production, such as increased expression of Tmem27, the proapoptotic thioredoxin-interacting protein Txnip, Cck and Pcsk2, and downregulation of Cacnald [23, 28-30].

Most strikingly, genes for OXPHOS were substantially upregulated by the HF, demonstrating enhanced oxidative metabolism in islets of animals receiving HF or highcarbohydrate diet. Moreover, the majority of enzymes for pyruvate metabolism and TCA cycle were significantly upregulated by the HF, suggesting an increased flux of fatty acid-derived acyl-CoA towards mitochondrial oxidation in the presence of dietary carbohydrates. Thus, increased availability of carbohydrates stimulates oxidative metabolism in islets, presumably by providing sufficient TCA intermediates in the mitochondrial matrix (anaplerosis).

Alterations in expression of OXPHOS genes has been regarded as a key change in the insulin-resistant state in human patients and animal models of type 2 diabetes [3133]. Since OXPHOS is a major source of reactive oxygen species (ROS) production, increased mitochondrial oxygen flux leads to increased formation of ROS [34-37]. ROS include highly reactive moieties, such as superoxide anions, hydrogen peroxide and hydroxyl radicals, which have been implicated in the pathogenesis of diabetes [38, 39]. Consistent with elevated ROS production in HF islets, the gene expression of regulators of the cellular redox state, including catalase, glutathione peroxidase 1, peroxiredoxins and thioredoxin interacting protein, was increased, strongly implicating ROS in the early state of beta cell failure. Proteomic analysis of isolated islets may be used to further investigate the diet-induced alterations in OXPHOS gene expression and protein modifications in response to increased oxidative stress.

Among the most differentially expressed genes in response to the dietary intervention were the transcription factor Chrebp and the peptide hormone Cck. Interestingly, cholecystokinin mRNA, which was upregulated about tenfold by the HF, appears to be highly enriched in LCM islets. Recent evidence suggests that cholecystokinin acts as a proliferative signal on beta cells [28], implicating it in the compensatory hyperplasia of islet cells in the prediabetic state. ChREBP has been described as a transcriptional sensor for glucose in the liver, which becomes activated upon glucose stimulation and triggers production of lipogenic enzymes for conversion of glucose into fat [24]. We confirmed ChREBP protein production in islets by immunohistochemistry (Fig. 4a-d). Moreover, Chrebp mRNA levels in islets and liver were comparable (Fig. 4e). Consistent with previous reports [24], HF-induced hyperglycaemia resulted in activation of ChREBP in the liver and subsequent induction of lipogenic enzymes, including acetyl-CoA carboxylase, fatty acid synthase, stearoyl-CoA desaturase 1 and liver-type pyruvate kinase (Fig. $4 \mathrm{e}$ and data not shown). Interestingly, in islets, ChREBP was considerably decreased in response to the diabetogenic HF, whereas transcription of ChREBP target genes was not affected, clearly indicating that in islets ChREBP controls different target genes than in the liver. In fact, a recent report demonstrated that ChREBP mediates glucose-induced expression of proapoptotic Txnip and induces $\mathrm{H} 4$ acetylation in beta cells, implicating a wider role of ChREBP beyond regulation of hepatic enzymes [40].

In summary, our data clearly demonstrate the critical role of dietary carbohydrates in the pathophysiology of HFinduced diabetes. LCM allows isolation of authentic pancreatic islet cells from their native tissue environment for genome-wide expression profiling. In islets, the diabetes-protective effect of a CHF diet correlates with reduced expression of genes for OXPHOS and antioxidants, suggesting a major contribution of ROS to dietinduced beta cell failure. Thus, our approach successfully identified candidate pathways and molecules that may play a role in the aetiology of type 2 diabetes.

Acknowledgements This work was supported in part by the European Union (EUGENE2, LSHM-CT-2004-512013; SysProt, LSHG-CT-2006-37457), the Gates Cambridge Trust and the UK Medical Research Council's Centre for Obesity and Related Metabolic Disorders (MRC-CORD).

Duality of interest The authors declare that there is no duality of interest associated with this manuscript.

Open Access This article is distributed under the terms of the Creative Commons Attribution Noncommercial License which permits any noncommercial use, distribution, and reproduction in any medium, provided the original author(s) and source are credited.

\section{References}

1. Doria A, Patti ME, Kahn CR (2008) The emerging genetic architecture of type 2 diabetes. Cell Metab 8:186-200 
2. Schmidt C, Gonzaludo NP, Strunk S et al (2008) A meta-analysis of QTL for diabetes-related traits in rodents. Physiol Genomics 34:42-53

3. Wuschke S, Dahm S, Schmidt C, Joost HG, Al-Hasani H (2007) A meta-analysis of quantitative trait loci associated with body weight and adiposity in mice. Int J Obes (Lond) 31:829-841

4. Yki-Jarvinen H (1992) Glucose toxicity. Endocr Rev 13:415-431

5. Poitout V, Robertson RP (2008) Glucolipotoxicity: fuel excess and beta-cell dysfunction. Endocr Rev 29:351-366

6. Stumvoll M, Goldstein BJ, van Haeften TW (2005) Type 2 diabetes: principles of pathogenesis and therapy. Lancet 365:1333-1346

7. Ahn YB, Xu G, Marselli L et al (2007) Changes in gene expression in beta cells after islet isolation and transplantation using laser-capture microdissection. Diabetologia 50:334-342

8. Marselli L, Thorne J, Ahn YB et al (2008) Gene expression of purified beta-cell tissue obtained from human pancreas with laser capture microdissection. J Clin Endocrinol Metab 93:1046-1053

9. Ruan H, Zarnowski MJ, Cushman SW, Lodish HF (2003) Standard isolation of primary adipose cells from mouse epididymal fat pads induces inflammatory mediators and down-regulates adipocyte genes. J Biol Chem 278:47585-47593

10. Jurgens HS, Neschen S, Ortmann S et al (2007) Development of diabetes in obese, insulin-resistant mice: essential role of dietary carbohydrate in beta cell destruction. Diabetologia 50:1481-1489

11. Livak KJ, Schmittgen TD (2001) Analysis of relative gene expression data using real-time quantitative PCR and the 2(-Delta Delta C(T)) Method. Methods 25:402-408

12. Gentleman RC, Carey VJ, Bates DM et al (2004) Bioconductor: open software development for computational biology and bioinformatics. Genome Biol 5:R80

13. Wu Z, Irizarry RA, Gentleman R, Martinez-Murillo F, Spencer F (2004) A model based background adjustment for oligonucleotide expression arrays. JASA 99:909-917

14. Dai M, Wang P, Boyd AD et al (2005) Evolving gene/transcript definitions significantly alter the interpretation of GeneChip data. Nucleic Acids Res 33:e175

15. Kanehisa M, Goto S (2000) KEGG: Kyoto encyclopedia of genes and genomes. Nucleic Acids Res 28:27-30

16. Ashburner M, Ball CA, Blake JA et al (2000) Gene ontology: tool for the unification of biology. The Gene Ontology Consortium. Nat Genet 25:25-29

17. HR KE, Kowald A, Wierling C, Lehrach H (2005) Systems biology in practice. Wiley-VCH, Weinheim

18. Cameron DP, Opat F, Insch S (1974) Studies of immunoreactive insulin secretion in NZO mice in vivo. Diabetologia 10(Suppl):649654

19. Saxena R, Voight BF, Lyssenko V et al (2007) Genome-wide association analysis identifies loci for type 2 diabetes and triglyceride levels. Science 316:1331-1336

20. Zeggini E, Weedon MN, Lindgren CM et al (2007) Replication of genome-wide association signals in UK samples reveals risk loci for type 2 diabetes. Science 316:1336-1341

21. Scott LJ, Mohlke KL, Bonnycastle LL et al (2007) A genomewide association study of type 2 diabetes in Finns detects multiple susceptibility variants. Science 316:1341-1345

22. Zeggini E, Scott LJ, Saxena R et al (2008) Meta-analysis of genome-wide association data and large-scale replication identifies additional susceptibility loci for type 2 diabetes. Nat Genet $40: 638-645$
23. Namkung Y, Skrypnyk N, Jeong MJ et al (2001) Requirement for the L-type $\mathrm{Ca}(2+)$ channel alpha(1D) subunit in postnatal pancreatic beta cell generation. J Clin Invest 108:1015-1022

24. Postic C, Dentin R, Denechaud PD, Girard J (2007) ChREBP, a transcriptional regulator of glucose and lipid metabolism. Annu Rev Nutr 27:179-192

25. Leiter EH, Coleman DL, Ingram DK, Reynolds MA (1983) Influence of dietary carbohydrate on the induction of diabetes in C57BL/KsJ-db/db diabetes mice. J Nutr 113:184-195

26. Plum L, Giesen K, Kluge R et al (2002) Characterisation of the mouse diabetes susceptibility locus Nidd/SJL: islet cell destruction, interaction with the obesity QTL Nob1, and effect of dietary fat. Diabetologia 45:823-830

27. Plum L, Kluge R, Giesen K, Altmuller J, Ortlepp JR, Joost HG (2000) Type 2 diabetes-like hyperglycemia in a backcross model of NZO and SJL mice: characterization of a susceptibility locus on chromosome 4 and its relation with obesity. Diabetes 49:1590 1596

28. Lavine JA, Raess PW, Davis DB et al (2008) Overexpression of pre-pro-cholecystokinin stimulates beta-cell proliferation in mouse and human islets with retention of islet function. Mol Endocrinol 22:2716-2728

29. Akpinar P, Kuwajima S, Krutzfeldt J, Stoffel M (2005) Tmem27: a cleaved and shed plasma membrane protein that stimulates pancreatic beta cell proliferation. Cell Metab 2:385-397

30. Chen J, Saxena G, Mungrue IN, Lusis AJ, Shalev A (2008) Thioredoxin-interacting protein: a critical link between glucose toxicity and beta-cell apoptosis. Diabetes 57:938-944

31. Sparks LM, Xie H, Koza RA et al (2005) A high-fat diet coordinately downregulates genes required for mitochondrial oxidative phosphorylation in skeletal muscle. Diabetes 54:1926-1933

32. Misu H, Takamura T, Matsuzawa N et al (2007) Genes involved in oxidative phosphorylation are coordinately upregulated with fasting hyperglycaemia in livers of patients with type 2 diabetes. Diabetologia 50:268-277

33. Takamura T, Misu H, Matsuzawa-Nagata N et al (2008) Obesity upregulates genes involved in oxidative phosphorylation in livers of diabetic patients. Obesity (Silver Spring) 16:2601-2609

34. Bonawitz ND, Chatenay-Lapointe M, Pan Y, Shadel GS (2007) Reduced TOR signaling extends chronological life span via increased respiration and upregulation of mitochondrial gene expression. Cell Metab 5:265-277

35. Houstis N, Rosen ED, Lander ES (2006) Reactive oxygen species have a causal role in multiple forms of insulin resistance. Nature 440:944-948

36. Osmundsen H, Bremer J, Pedersen JI (1991) Metabolic aspects of peroxisomal beta-oxidation. Biochim Biophys Acta 1085:141-158

37. Brownlee M (2001) Biochemistry and molecular cell biology of diabetic complications. Nature 414:813-820

38. Furukawa S, Fujita T, Shimabukuro M et al (2004) Increased oxidative stress in obesity and its impact on metabolic syndrome. J Clin Invest 114:1752-1761

39. Urakawa H, Katsuki A, Sumida Y et al (2003) Oxidative stress is associated with adiposity and insulin resistance in men. $\mathrm{J}$ Clin Endocrinol Metab 88:4673-4676

40. Cha-Molstad H, Saxena G, Chen J, Shalev A (2009) Glucosestimulated expression of TXNIP is mediated by CHREBP, p300 and histone $\mathrm{H} 4$ acetylation in pancreatic beta cells. J Biol Chem $284: 16898-16905$ 